\title{
Invisible decays of low mass Higgs bosons in supersymmetric models
}

\author{
P. N. Pandita, ${ }^{1}$ Monalisa Patra ${ }^{2}$ \\ 1 P-30, North Eastern Hill University, Shillong 793 002, India \\ ${ }^{2}$ Department of Theoretical Physics, Tata Institute of Fundamental Research, Mumbai 400 005, India
}

\begin{abstract}
The discovery of a $126 \mathrm{GeV}$ Higgs like scalar at the LHC along with the non observation of the supersymmetric particles, has in turn lead to constraining various supersymmetric models through the Higgs data. We here consider the case of both the minimal supersymmetric standard model (MSSM), as well its extension containing an additional chiral singlet superfield, the next-to-minimal or nonminimal supersymmetric standard model (NMSSM). A lot of work has been done in the context of the lightest scalar of these models being identified as the $126 \mathrm{GeV}$ state discovered at the LHC. We here however concentrate on the case where we identify the second lightest Higgs boson as the $126 \mathrm{GeV}$ state discovered at the CERN LHC and consider the invisible decays of the low mass Higgs bosons in both MSSM and NMSSM. In case of the MSSM, we consider $H \approx 126 \mathrm{GeV}$ and $h \approx 98$ $\mathrm{GeV}$, known as the non-decoupling regime, whereas in case of the NMSSM $h_{2} \approx 126 \mathrm{GeV}$, with $m_{h_{1}}$ and $m_{a_{1}}$ varying depending on the parameter space. We find that in case of the MSSM with universal boundary conditions at the GUT scale, it is not possible to have light neutralinos leading to the decay channel $H \rightarrow \tilde{\chi}_{1}^{0} \tilde{\chi}_{1}^{0}$. The invisible decay mode is allowed in case of certain $S O(10)$ and $E_{6}$ grand unified models with large representations and nonuniversal gaugino masses at the GUT scale. In case of the NMSSM, for the parameter space considered it is possible to have the invisible decay channel with universal gaugino masses at the GUT scale. We furthermore consider the most general case, with $M_{1}$ and $M_{2}$ as independent parameters for both MSSM and NMSSM. We isolate the regions in parameter space in both cases, where the second lightest Higgs boson has a mass of $126 \mathrm{GeV}$ and then concentrate on the invisible decay of Higgs to lighter neutralinos. The other non-standard decay mode of the Higgs is also considered in detail. The invisible Higgs branching ratio being constrained by the LHC results, we find that in this case with the second lightest Higgs being the $126 \mathrm{GeV}$ state, more data from the LHC is required to constrain the neutralino parameter space, compared to the case when the lightest Higgs boson is the $126 \mathrm{GeV}$ state.
\end{abstract}

PACS numbers: $12.60 . \mathrm{Jv}, 14.80 . \mathrm{Da}, 14.80 . \mathrm{Nb}$

\section{INTRODUCTION}

A new era of particle physics has begun with the discovery of the neutral scalar by the ATLAS [1] and CMS [2] collaborations. It is entirely likely that this state is the long sought after Higgs boson of the standard model (SM) and is being pursued as a main window for new physics searches, Though the recent results are already pointing towards a SM like Higgs, final conclusion can only be drawn through a detailed study of the properties of the new boson. These studies will indicate, whether the decay widths of the particle are in accordance with the predictions of the SM or of its extensions. Popular among the latter are the Minimal Supersymmetric Standard Model (MSSM) and the Next-to-Minimal Supersymmetric Model (NMSSM). The Higgs sector in MSSM consists of five physical Higgs which includes two CP even Higgs $(h, H)$, one CP odd Higgs $A$ and a pair of charged Higgs $\left(H^{ \pm}\right)$. In case of NMSSM, the $\mu$ parameter of MSSM, is replaced by $\lambda\langle S\rangle$, which is generated from a trilinear superpotential coupling $\lambda H_{1} H_{2} S$, when $S$ obtains a vacuum expectation value $\langle S\rangle$. Here $H_{1}$ and $H_{2}$ are the two Higgs doublet, whereas $S$ is the chiral singlet superfield. This term in turn leads to three CP-even Higgs bosons, $h_{1,2,3}$, two CP-odd Higgs bosons, $a_{1,2}$, and a pair of charged Higgs bosons, $H^{ \pm}$.

The MSSM as well as the NMSSM predict the existence of a dark matter candidate, which in large parts of the parameter space is a neutralino. If the neutralino is sufficiently light, Higgs decay to neutralinos will be kinematically allowed. Such a light neutralino with the required relic density is still supported by the recent experimental results [3-6]. The presence of light neutralino therefore has implications on the Higgs phenomenology, as it gives rise to the decay channel $h \rightarrow \tilde{\chi_{1}^{0}} \tilde{\chi_{1}^{0}}$, i.e. invisible branching ratio. With the latest experimental results, fits are being performed to check how much deviation is allowed by the recent data, in order to take into account new physics scenarios. The invisible Higgs decay width has been constrained by various groups by performing fits of the signal strengths in various search channels using the latest LHC Higgs data. The results of some of the most recent global fits:

1. Considering the Higgs couplings to the quarks, leptons and vector bosons to be free, $\mathrm{BR}\left(h \rightarrow \tilde{\chi}_{1}^{0} \tilde{\chi}_{1}^{0}\right)<0.16(0.38)$ at $68 \%(95 \%) \mathrm{CL}[7]$.

2. With the assumption that the Higgs coupling to fermions and gauge bosons are SM like, and the only 
new physics is from the Higgs invisible decay width, $\operatorname{BR}\left(h \rightarrow \tilde{\chi}_{1}^{0} \tilde{\chi}_{1}^{0}\right)<0.52$ at $68 \%$ CL $[8]$.

Direct search for invisible decaying Higgs produced in association with a $Z$ boson has been carried out by the ATLAS and CMS collaborations in LHC. They have, in turn, placed limits on the branching fraction of the Higgs boson to invisible particles, with the branching fraction greater than $65 \%$ and $75 \%$ excluded at $95 \%$ CL by ATLAS [9, 10] and CMS [11] respectively. The CMS collaboration has also carried out a similar search for invisible branching ratio of the Higgs boson produced in the vector boson fusion process and have placed an upper limit of $69 \%$ [12]. ${ }^{1}$

Direct searches for SUSY particles at the LHC so far have come empty handed. Furthermore, several analyses based on simple versions of the MSSM and other models have ruled out significant regions of the parameter space. However, the parameter space under more general assumptions still remains largely unexplored. One possibility of exploring these regions is to ask under what conditions the $126 \mathrm{GeV}$ state corresponds to the neutral higgs particles in the spectrum of the model. Popular among these is the case where the lightest Higgs boson of MSSM $(h)$ and NMSSM $\left(h_{1}\right)$ is identified with the state discovered at LHC at $126 \mathrm{GeV}$ and has been studied in great detail. In [13], the authors studied the decay of this lightest Higgs boson into neutralinos in these low energy supersymmetric models. The neutralino sector of these models were then constrained, from the limits on the invisible decay width. The regions of the parameter space where the lightest Higgs boson has a mass of around $126 \mathrm{GeV}$ was isolated and then the regions where this Higgs can decay into light neutralinos were studied in details. It was found that it was not possible to have a massless neutralino in MSSM both in case of universal and nonuniversal gaugino masses at the GUT scale, except for some higher representation of $E_{6}$. In case of NMSSM although it was possible to have a massless neutralino with universal gaugino masses at the GUT scale, it was not possible to obtain $M_{h_{1}}=126 \mathrm{GeV}$ and simultaneously have massless neutralino or $M_{\tilde{\chi}_{1}^{0}} \leq M_{h_{1}} / 2$. The results were therefore obtained by considering the soft gaugino masses $M_{1}$ and $M_{2}$ as two independent parameters, unconstrained by grand unification. In case of NMSSM, for certain regions of the parameter space there were additional nonstandard decay channels like $h_{1} \rightarrow a_{1} a_{1}, Z a_{1}$. The composition of the $\tilde{\chi}_{1}^{0}$ was important in determining the invisible branching ratio, with some regions of the parameter space allowing large invisible branching ratio.

Note that there are no a priori reasons to believe that the $126 \mathrm{GeV}$ boson is the lightest Higgs boson. In the MSSM either $h$ and $H$ can be identified with the discovered $126 \mathrm{GeV}$ boson, with either $h \approx 126 \mathrm{GeV}$, $H \geq 800 \mathrm{GeV}$ or $H \approx 126 \mathrm{GeV}, M_{h}<M_{H}$. Analogously for the NMSSM there can be many choices, $(a) h_{1} \approx$ $126 \mathrm{GeV}$, and $M_{h_{2,3}}>M_{h_{1}}(b) h_{2} \approx 126 \mathrm{GeV}$, and $M_{h_{3}}>M_{h_{2}}, M_{h_{1}}<M_{h_{2}}$. The mass of the CP-odd Higgs Bosons varies in the range of $4 \mathrm{GeV}$ to $\mathrm{TeV}$ (to be discussed later), whereas the charged Higgs boson are very massive with masses of the order $1 \mathrm{TeV}$. This scenario with $M_{H} \approx 126 \mathrm{GeV}$ is mainly in light of the observed LEP excess [14] in the $e^{+} e^{-} \rightarrow Z h \rightarrow Z b \bar{b}$ channel around $M_{b \bar{b}} \approx 100 \mathrm{GeV}$, which indicates that there may be a lighter Higgs boson less than $100 \mathrm{GeV}$.

Here we take this possibility seriously and ask under what circumstances this is realized, and to what extent the measured properties allow this scenario to survive. Crucial to this is the possibility that the uncertainty in the width is saturated by invisible decays, rendered possible when there are states lighter than $63 \mathrm{GeV}$ in the spectrum. In this work we firstly delineate regions of the parameter space, which give rise to two light Higgs and then study in details the branching ratio of the $126 \mathrm{GeV}$ Higgs to non standard SM particles. This second lightest $126 \mathrm{GeV}$ Higgs can decay to a pair of lightest neutralinos as well as to a pair of lightest Higgs in some regions of parameter space. We consider both the cases here. Moreover in case of NMSSM, the decay of $h_{2}$ to a pair of $a_{1}$ is also kinematically allowed in some regions. Considering the limit on the invisible branching ratio from the experiments and global fits, the neutralino sector of these supersymmetric models are then constrained accordingly. In most studies, the parameter space of the models are constrained with the assumption of the universality of the gaugino masses at the GUT scale. Note the gaugino masses need not be universal at the GUT scale. This happens when the SM gauge group is embedded in a grand unified gauge group. The phenomenology of the neutralinos at the weak scale, is then affected via the renormalization group evolution of these gaugino mass parameters. At the weak scale, there will be a possibility of massless neutralinos [15] depending on the gaugino masses at the GUT scale. We find that for the parameter space which allows a 126 $\mathrm{GeV}$, the second lightest Higgs boson in MSSM, analogous to the case where the $126 \mathrm{GeV}$ state was identified with the lightest Higgs boson, it is not possible to have a massless neutralino with universal gaugino mass parameters $M_{1}$ and $M_{2}$. The result holds even for nonuniversal gaugino mass parameters except for a higher dimensional representation of $E_{6}$. Nevertheless, the decay of Higgs to lightest neutralinos $\left(M_{\tilde{\chi}_{1}^{0}} \leq M_{H} / 2\right)$

\footnotetext{
${ }^{1}$ In our analysis we will use the most stringent limit obtained on the invisible branching ratio to be less than $38 \%$ [7].
} 
is allowed for some representations in case of MSSM with nonuniversal gaugino masses at the GUT scale. Analogous in the case of the NMSSM, it is not possible to obtain $M_{h_{2}}=126 \mathrm{GeV}$ and simultaneously have massless neutralinos, with universal gaugino masses at the GUT scale but the decay of the Higgs $\left(h_{2}\right)$ to the lightest neutralinos is allowed $\left(M_{\tilde{\chi}_{1}^{0}} \neq 0\right.$ and $\left.M_{\tilde{\chi}_{1}^{0}} \leq M_{h_{2}} / 2\right)$. This assumption of the GUT relation between $M_{1}$ and $M_{2}$, is biased to a particular scenario, so we do not consider the universality assumption on the gaugino mass parameters and rather treat $M_{1}, M_{2}$ as two independent parameters.

In view of the considerations above, we have now considered the possibility of the second lightest neutral Higgs to be the $126 \mathrm{GeV}$ state discovered by the LHC, in some versions of the MSSM and the NMSSM along with its invisible decays due to the presence of light neutralinos or other light states present in the spectrum. Thus, the outline of the draft is as follows. In Sec. II we study the existence of a massless neutralino or a neutralino with mass less than the half the mass of $126 \mathrm{GeV}$ Higgs boson in the context of MSSM, with appropriate boundary conditions as dictated by grand unification based on $S U(5), S O(10)$ and $E_{6}$ gauge groups. The relevant experimental constraints on the lightest Higgs $\left(m_{h}<m_{H}\right.$, with $\left.m_{H} \approx 126 \mathrm{GeV}\right)$ as well as other SUSY particles is considered. The decay of the lightest as well as the second lightest Higgs to neutralinos is considered in SubSec. II A. The Higgs sector in case of NMSSM is investigated in detail in Sec. III. We first isolate the parameter space which supports $h_{2}$ in the appropriate mass window $123-127 \mathrm{GeV}$. Then the invisible decay of the second lightest Higgs boson along with the other nonstandard decay modes is considered in detail. The dominant decay mode of the lightest Higgs boson $h_{1}$ is also considered in this section. Finally, we summarize our results in Sec. IV.

\section{HIGGS AND NEUTRALINO SECTOR IN MSSM}

Let us begin by recalling that the Higgs sector in MSSM has five physical mass eigenstates, two CP even and one $\mathrm{CP}$ odd neutral along with a pair of charged scalar bosons. The Higgs spectrum at tree level is completely determined by two independent parameters $M_{A}$ and $\tan \beta$, where $\tan \beta$ is the ratio of the vacuum expectation value of the Higgs field and $M_{A}$ is the mass of the pseudoscalar Higgs. In addition to this, the MSSM Higgs sector also depends on the stop masses along with the stop mixing parameter $X_{t}$, when the radiative corrections are taken into account. Here $X_{t}=A_{t}-\mu / \tan \beta$, with $A_{t}$ as the trilinear Higgs-stop coupling and $\mu$ is the Higgsino mass parameter. The Higgs sector of the MSSM, with a Higgs Boson of mass $\approx 126 \mathrm{GeV}$, and with SM like cross sections and branching fractions, can be broadly divided into two distinct regimes depending on the magnitude of $M_{A}$.

- The decoupling regime, where $M_{A} \gg M_{Z}$. In this case the lightest CP even boson $h$ has mass around $126 \mathrm{GeV}$, whereas all the others $H, A$ and $H^{ \pm}$are almost degenerate and have mass equal to $M_{A}$.

- The non-decoupling regime is the one where $M_{A} \leq 130 \mathrm{GeV}$. The heavy $\mathrm{CP}$ even state $H$ is $\mathrm{SM}$ like, whereas the other neutral bosons are almost degenerate in mass $M_{h} \equiv M_{A}$. The mass of $h$ and $A$ can vary from the $Z$ boson mass to the heavy neutral state $H$, depending on the value of $M_{A}$ and $\tan \beta$. The charged state $H^{ \pm}$will be slightly heavier, but still light enough to be detected in the Large Hadron Collider. Moreover in this regime, with the mass of $H$ being around $126 \mathrm{GeV}$, the mass of the other gauge bosons like $h, A$ and $H^{ \pm}$are bounded from above.

The LEP collaborations have placed lower bounds on the masses of the neutral Higgs Boson $M_{A}$ and the lightest scalar $h$ [16]. The lower bounds on $M_{h}$ and $M_{A}$ are usually obtained from the upper bound on the cross section $\sigma\left(e^{+} e^{-} \rightarrow Z h\right)$ and $\sigma\left(e^{+} e^{-} \rightarrow A h\right)$. This in turn has led to values of $M_{h}$ and $M_{A}$ less than $92.9 \mathrm{GeV}$ and $93.4 \mathrm{GeV}$ being excluded at $95 \%$ C.L. Along with it values of $\tan \beta$ between 0.7 and 2 are also excluded. Recent searches of the extra Higgs boson at the LHC have put new bounds on $\tan \beta$ as a function of $M_{A}$ [17-20]. CMS data [19] has excluded regions of $\tan \beta$ above 6 for $M_{A}$ below $250 \mathrm{GeV}$ in the $m_{h}^{\max }$ scenario.

The main focus in this present work will be on the non-decoupling regime, and as a result, we would like to make some observations on the value of $M_{A}$ and $\tan \beta$ chosen for our analysis. Since this regime is mainly characterized by the pseudoscalar mass being less than $150 \mathrm{GeV}$, we plot in Fig. 1 the MSSM Higgs boson mass as a function of $M_{A}$, for two different values of $\tan \beta$. The other SUSY parameter $A_{t}$, which affects the Higgs sector is fixed assuming maximal stop mixing. It can be seen from the left hand side of Fig. 1 , in case of tan $\beta$ $=10$, for $M_{A} \leq 120 \mathrm{GeV}, M_{H}$ is around $126 \mathrm{GeV}$, whereas $M_{A}$ and $M_{h}$ are almost degenerate. The maximal value of $M_{A}$ which allows for $M_{H}$ around $126 \mathrm{GeV}$ is $130 \mathrm{GeV}$. The right hand side of Fig. 1 shows the masses of the Higgs bosons as a function of $M_{A}$ for $\tan \beta=40$. We see that for our choice of SUSY parameters, for $M_{A}$ around $125 \mathrm{GeV}$, the three neutral Higgs bosons have comparable masses, $M_{H} \simeq M_{h} \simeq M_{A}$. This special case where the Higgs masses are close to each other, is called the intense coupling scenario. The LHC phenomenology of this scenario has been studied in details in the past [21-23]. It has been known that in 

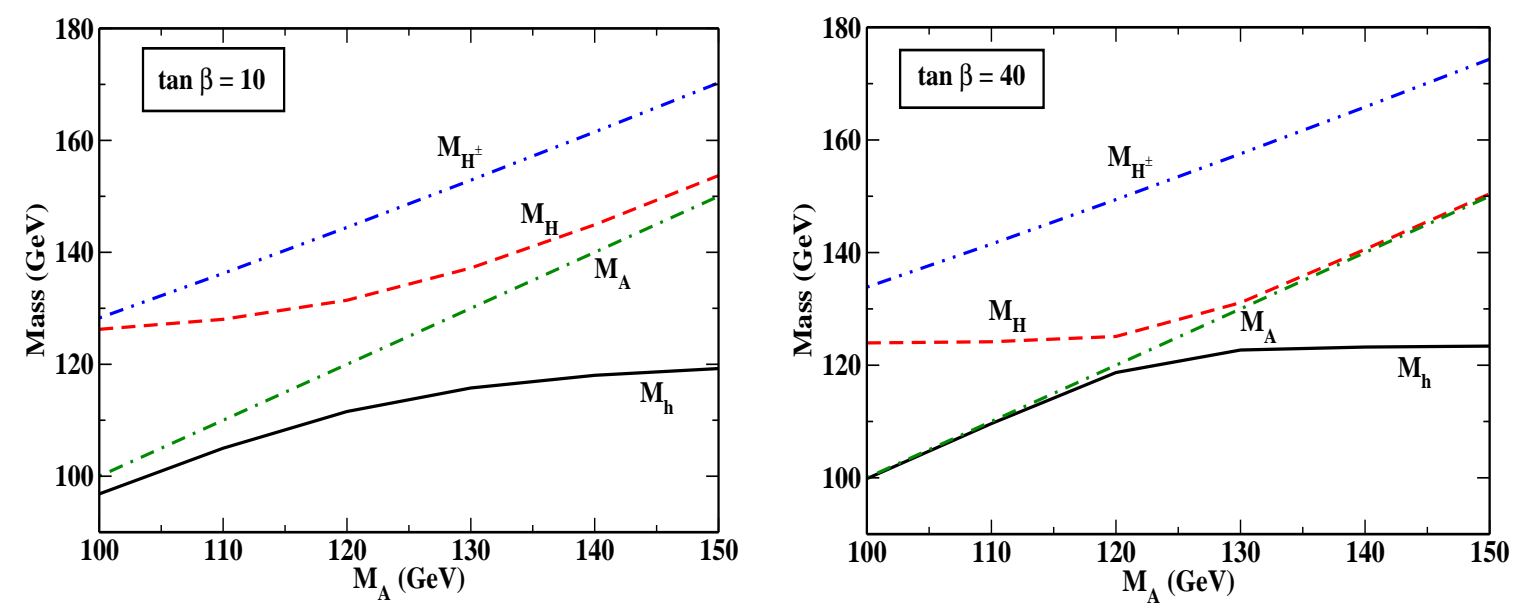

FIG. 1: The masses of MSSM Higgs bosons as a function of $M_{A}$ for two distinct values of $\tan \beta$.

this intense coupling scenario the neutral bosons $(h, H)$ couplings to the gauge bosons are suppressed with respect to the SM, since $A$ does not couple to the gauge bosons. Furthermore, the neutral bosons in this case will mainly decay to the down type fermions, due to the enhancement of its coupling. The recent LHC results being favoring a SM like Higgs along with large values of $\tan \beta$ being disfavored by the CMS data, we have concentrated on the case with $M_{A} \approx 100 \mathrm{GeV}$ and small values of $\tan \beta$, in order to have the cross section times branching ratio of the Higgs to any SM particle in agreement with the recent LHC results. The value of $\tan \beta$ is tuned along with the parameter $A_{t}$, so that $M_{H}$ is in the range $124<M_{H}<127 \mathrm{GeV}$, and $M_{h} \simeq 97$ $\mathrm{GeV}$.

In the past, various studies have shown that certain regions of the parameter space of MSSM, allow a Higgs boson with mass $126 \mathrm{GeV}$ both in the decoupling and non-decoupling regime, satisfying the LHC constraints. For most of the allowed parameter space, the Higgs decay to the lightest neutralinos is kinematically allowed, leading to invisible decay modes. It will therefore be very important to study the couplings of the newly discovered particle at high precision. As mentioned before global fits have been performed on the couplings of the newly discovered particle, in order to place upper bounds on the invisible decay width. Taking into account these bounds, the parameter space of these new physics scenarios can be further constrained, since the regions giving a large invisible Higgs decay branching ratio will be in conflict with the experiments. This was earlier done in the context of MSSM, in the decoupling scenario, where it was found that large regions in $\mu-M_{1}$ parameter space was disfavored [24] by the bounds on the invisible Higgs decay width, for different values of $\tan \beta$. In this work, we have investigated further to see whether the same result holds in the non-decoupling scenario, assuming $H$ to be the $126 \mathrm{GeV}$ boson.

Before proceeding further we give a brief review of the neutralino sector in the MSSM. The physical mass eigenstates are obtained after the electroweak symmetry breaking, from the diagonalization of the neutralino mass matrix [25, 26], with the neutralinos being an admixture of the fermionic partners of the two Higgs doublets, $H_{1}$ and $H_{2}$, and the fermionic partners of the neutral gauge bosons.

$$
M_{\mathrm{MSSM}}=\left(\begin{array}{cccc}
M_{1} & 0 & -M_{Z} \sin \theta_{W} \cos \beta & M_{Z} \sin \theta_{W} \sin \beta \\
0 & M_{2} & M_{Z} \cos \theta_{W} \cos \beta & -M_{Z} \cos \theta_{W} \sin \beta \\
-M_{Z} \sin \theta_{W} \cos \beta & M_{Z} \cos \theta_{W} \cos \beta & 0 & -\mu \\
M_{Z} \sin \theta_{W} \sin \beta & -M_{Z} \cos \theta_{W} \sin \beta & -\mu & 0
\end{array}\right),
$$

where $M_{1}$ and $M_{2}$ are the $U(1)_{Y}$ and the $S U(2)_{L}$ soft supersymmetry breaking gaugino mass parameters, $\mu$ is the Higgs(ino) mass parameter, $M_{Z}$ is the $Z$ boson mass, $\theta_{W}$ is the weak mixing angle and $\tan \beta=v_{2} / v_{1}$ is the ratio of the vacuum expectation values of the neutral components of the two Higgs doublet fields $H_{1}$ and $\mathrm{H}_{2}$. Since we are concentrating on the Higgs invisible decay mode, the light neutralino eigenstate of the neutralino mass matrix (II.1) is favorable. Therefore we consider the limiting case of the massless neutralino, which, at the tree level, arises when the determinant of the mass matrix (II.1) is zero. This in turn leads to the condition $[15]$

$$
\mu\left[M_{Z}^{2} \sin 2 \beta\left(M_{1} \cos ^{2} \theta_{W}+M_{2} \sin ^{2} \theta_{W}\right)-M_{1} M_{2}\right]=0
$$


The chargino mass lower bounds from the LEP experiments [30], excludes the solution $\mu=0$,

$$
|\mu|, \quad M_{2} \geqslant 100 \mathrm{GeV} .
$$

Therefore the other possible solution to (II.2) is

$$
M_{1}=\frac{M_{2} M_{Z}^{2} \sin ^{2} \theta_{W} \sin 2 \beta}{\mu M_{2}-M_{Z}^{2} \cos ^{2} \theta_{W} \sin 2 \beta} .
$$

In order to get a massless neutralino, for fixed values of $\mu, M_{2}$ and $\tan \beta$, one can find a value of $M_{1}$ consistent with (II.4).

In the earlier work [13] it was found that it is not possible to have a massless neutralino, both with the gaugino parameters being universal and non universal at the GUT scale, except for some higher representation of $E_{6}$. The light neutralino with mass less than half the mass of the Higgs boson, is still not ruled out by the current experiments. It is seen that for the models, with the ratio of $M_{1} / M_{3}<1 / 28$, the invisible decay of Higgs to the lightest neutralinos is allowed and holds true for both the coupling and the non-decoupling regimes of MSSM. This is mainly by taking into account the constraint on the gluino mass $\left(M_{\tilde{g}} \approx M_{3}\right)>$ $1.3 \mathrm{TeV}$ from the LHC experiments, and the other gaugino mass parameters being relatively fixed from the boundary conditions at the electroweak scale. As can be seen from Ref. [13], this condition on the ratio $M_{1} / M_{3}$ is satisfied by some of the higher dimensional representation of $S O(10)$ and $E_{6}$.

\section{A. Decay of Higgs to Neutralinos in the MSSM}

In this section we mainly concentrate on constraining the Higgs parameter space in case of MSSM, from the Higgs invisible decay width. One of the main assumptions that go into limiting the parameter space of these models is the universality of the gaugino mass parameters at the GUT scale. The LEP constraint on the charginos, has led to lower bound on the lightest neutralino mass

$$
M_{\tilde{\chi}_{1}^{0}}>46 \mathrm{GeV}
$$

at $95 \%$ C.L. in the context of the MSSM, assuming universal gaugino masses at the GUT scale [27]. The gaugino mass parameters need not be universal at the GUT scale, therefore the phenomenology of the neutralinos in all these cases will be affected depending on the renormalization group evolution of the gaugino mass parameters. We do not consider any specific representations, but instead consider a more generic case with $M_{1}$ and $M_{2}$ as independent parameters, in the non-decoupling scenario. With this consideration the lightest neutralino so obtained will be bino like, because the chargino mass bounds from LEP has already set lower limits on $M_{2}$ and $\mu$.

In MSSM, the decay width of the CP even neutral scalar bosons, to a pair of lightest neutralinos can be written as [28]

$$
\begin{aligned}
\Gamma\left(h \rightarrow \tilde{\chi}_{1}^{0} \tilde{\chi}_{1}^{0}\right) & =\frac{G_{F} M_{W}^{2} M_{h}}{2 \sqrt{2} \pi}\left(1-4 M_{\tilde{\chi}_{1}^{0}}^{2} / M_{h}^{2}\right)^{3 / 2}\left[\left(Z_{12}-\tan \theta_{W} Z_{11}\right)\left(Z_{13} \sin \alpha+Z_{14} \cos \alpha\right)\right]^{2}, \\
\Gamma\left(H \rightarrow \tilde{\chi}_{1}^{0} \tilde{\chi}_{1}^{0}\right) & =\frac{G_{F} M_{W}^{2} M_{H}}{2 \sqrt{2} \pi}\left(1-4 M_{\tilde{\chi}_{1}^{0}}^{2} / M_{H}^{2}\right)^{3 / 2}\left[\left(Z_{12}-\tan \theta_{W} Z_{11}\right)\left(Z_{13} \cos \alpha-Z_{14} \sin \alpha\right)\right]^{2},
\end{aligned}
$$

where $Z_{i j}$ are the elements of the matrix $Z$ which diagonalizes the neutralino mass matrix, and $\alpha$ is the mixing angle in the $C P$ even Higgs sector. The above (II.6), (II.7) shows that the invisible branching ratio requires $\tilde{\chi}_{1}^{0}$ to be a mixed state, with both gaugino and higgsino contribution. The invisible decay of the Higgs though favoured by a large higgsino fraction neutralino, will be mainly constrained by the $Z$ invisible decay width. The $Z$ width to a pair of lightest neutralinos is given by [29]

$$
\Gamma\left(Z \rightarrow \tilde{\chi}_{1}^{0} \tilde{\chi}_{1}^{0}\right)=\frac{G_{F} M_{Z}^{3}}{6 \sqrt{2} \pi}\left(1-4 M_{\tilde{\chi}_{1}^{0}}^{2} / M_{Z}^{2}\right)^{3 / 2}\left(Z_{13}^{2}-Z_{14}^{2}\right) .
$$

The invisible decay width of $Z$ to a pair of lightest neutralinos is restricted to

$$
\Gamma\left(Z^{0} \rightarrow \tilde{\chi}_{1}^{0} \tilde{\chi}_{1}^{0}\right)<3 \mathrm{MeV} .
$$

at $95 \%$ C.L. by the LEP collaborations [30]. 
The mass bound on the lightest chargino $M_{\tilde{\chi}^{ \pm}}>94 \mathrm{GeV}$ from the LEP experiments is taken into account [27]. The results are presented for a fixed value of $M_{2}=200 \mathrm{GeV}$, with the parameters $\mu$ and $M_{1}$ being varied. The other SUSY parameters like the squarks and gluinos are fixed to masses around $1 \mathrm{TeV}$ in accordance with the latest LHC results. The masses of the sleptons are taken to be greater than $500 \mathrm{GeV}$. Since we are considering the Higgs decay to the lightest neutralino pair, which are also one of the leading dark matter candidates, it will be necessary to check whether the kinematically allowed parameter region in the $\mu-M_{1}$ plane also gives the correct relic density as measured from WMAP [31, 32], i.e. $0.0925<\Omega h^{2}<0.1287$. In this work, the computation of the relic density has been performed with micrOMEGAs 3.2 [33], along with the production and decays of the SUSY particles being computed with CalcHEP [34].

Apart from the LHC constraints, the constraints from $(g-2)$ of the muon and other flavour constraints such as $b \rightarrow s \gamma$ and $B_{s} \rightarrow \mu^{+} \mu^{-}$are also taken into account, which are implemented within CalcHEP.
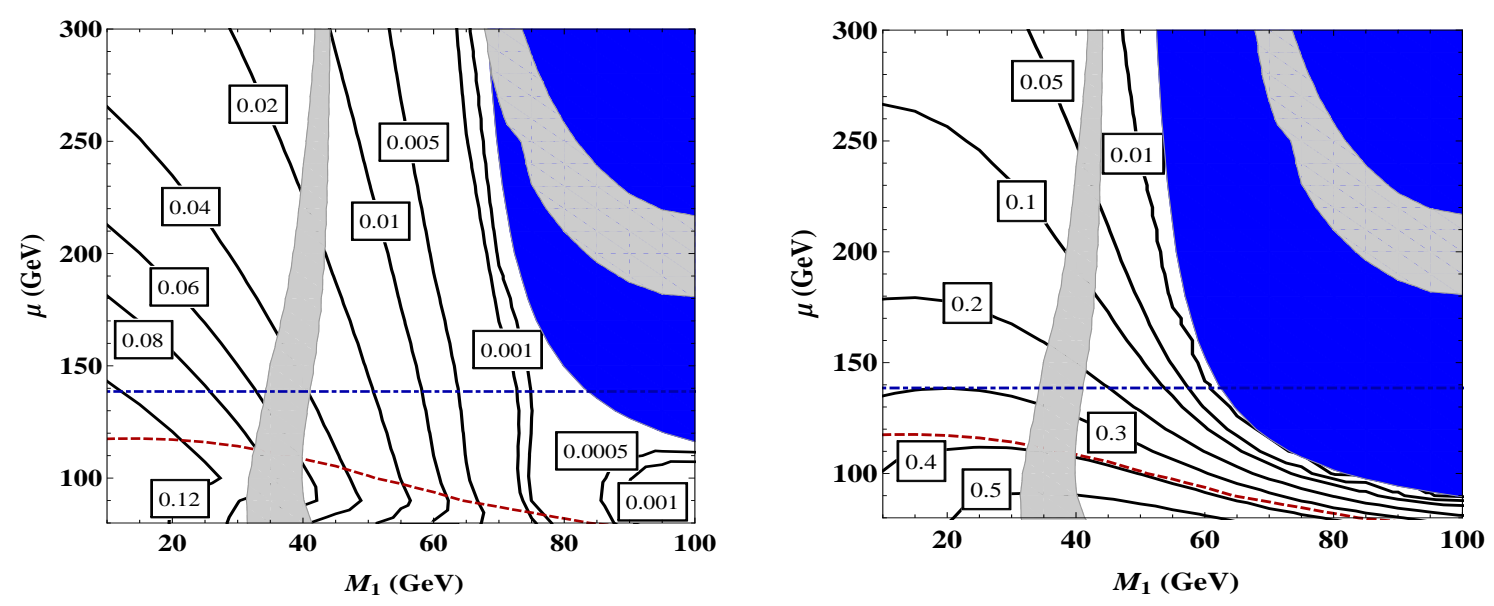

FIG. 2: The contours of constant branching ratio of $\left(H \rightarrow \tilde{\chi}_{1}^{0} \tilde{\chi}_{1}^{0}\right)$ (left) and $\left(h \rightarrow \tilde{\chi}_{1}^{0} \tilde{\chi}_{1}^{0}\right)$ (right) for a fixed value of tan $\beta$ $=6$ and $M_{2}=200 \mathrm{GeV}$. The region shaded in Blue is the region kinematically not allowed, whereas the Grey shaded region is the one with the relic density within the experimental limits.

We show in Fig. 2, the branching ratio of both CP even Higgs ( $H$ (left), $h$ (right)) to the lightest neutralinos. The regions with large $\mu$ and $M_{1}$ values as expected give rise to massive neutralinos, with $M_{\tilde{\chi}_{0}^{1}}>M_{H, h} / 2$, and is therefore kinematically not allowed and is shaded in Blue. The neutralino obtained by considering $M_{1}$ and $M_{2}$ as independent parameters is mostly a bino like as the LEP mass bound on chargino has already placed lower bound on $M_{2}$ and $\mu$. In the bino limit, the process which mainly contributes to the relic density is the one mediated by a $t$ - channel sfermion. We have calculated the relic density for different slepton masses varying from 150-1000 GeV. There being no significant change in the allowed parameter space, we have presented the results for sfermion mass of $500 \mathrm{GeV}$. It is seen that for most of the allowed parameter region of $\mu-M_{1}$, taking into account the LEP bound of the chargino mass [Blue-DotDashed] and the invisible decay width of the $Z$ boson [Red-Dashed], the invisible branching ratio of the Higgs $H$ is still too small to be probed at the LHC. The area below the Blue-DotDashed and Red-Dashed lines, are excluded from the LEP bound of the chargino and the invisible $Z$ decay width respectively. The parameter space can not be constrained by the latest limits on the invisible decay width from the LHC fits. This is in contrast to the situation $M_{h} \approx 126 \mathrm{GeV}$, where most of the $\mu-M_{1}$ parameter space was constrained from the bounds on the invisible branching ratio of the Higgs (h) $[13,24]$. Moreover in the non-decoupling scenario as can be seen from the right hand side of Fig. 2, the $\operatorname{BR}\left(h \rightarrow \tilde{\chi}_{1}^{0} \tilde{\chi}_{1}^{0}\right)$ is small compared to the decoupling case, due to an enhanced coupling to the $b$ quarks. The enhancement is mainly due to the $\sin \alpha$ term, in the coupling of $h$ to a pair of $b$ quarks, which is sensitive to the parameter $M_{A}$.

The shape of the contours in the left plot of Fig. 2, can be understood from the fact that, the $\operatorname{BR}\left(H \rightarrow \tilde{\chi}_{1}^{0} \tilde{\chi}_{1}^{0}\right)$ decreases for increasing $\mu$, due to the increase in neutralino mass. The dip in the contours for $\mu$ around 100 $\mathrm{GeV}$ is due to the fact that for a particular value of $M_{1}$, after $\mu$ decreases to a certain value, the other decay modes of Higgs such as $h \rightarrow \tilde{\chi}_{1}^{0} \tilde{\chi}_{2}^{0}, \tilde{\chi}_{1}^{0} \tilde{\chi}_{3}^{0}, \tilde{\chi}_{1}^{+} \tilde{\chi}_{1}^{-}$open up, leading to a decrease in the invisible BR. Most of the parameter space for $\mu<140 \mathrm{GeV}$, is however excluded by the chargino mass bound of $110 \mathrm{GeV}$. The same argument holds for $h$, the right plot of Fig. 2. We finally list in Table I the branchings of $h, H$ to different final states for our parameter choice of $M_{A}=105 \mathrm{GeV}, \tan \beta=6, M_{2}=100 \mathrm{GeV}, M_{1}=50 \mathrm{GeV}$ and $\mu=130$ $\mathrm{GeV}$. The BR of decay to neutralinos changes with the change of $\mu$ and $M_{1}$ as discussed before. 


\begin{tabular}{||c|c|c||}
\hline final states & $H$ branchings & $h$ branchings \\
\hline \hline & $M_{H}=125 \mathrm{GeV}$ & $M_{h}=97 \mathrm{GeV}$ \\
\hline \hline$l, L(e, \mu, \tau)$ & 0.089 & 0.074 \\
$b b$ & 0.841 & 0.735 \\
$c c$ & 0.004 & $3.5 \times 10^{-4}$ \\
$G G$ & 0.016 & $1.1 \times 10^{-3}$ \\
$A A$ & $1.7 \times 10^{-4}$ & $3.3 \times 10^{-5}$ \\
$W^{+} W^{-}$ & 0.024 & $6.5 \times 10^{-5}$ \\
$Z Z$ & $2.9 \times 10^{-3}$ & \\
$\tilde{\chi}_{1}^{0} \tilde{\chi}_{1}^{0}$ & 0.023 & 0.191 \\
\hline
\end{tabular}

TABLE I: Branching ratios of both $h, H$ to various decay channels, with our parameter choice of $M_{A}=105 \mathrm{GeV}$, tan $\beta$ $=6, M_{2}=200 \mathrm{GeV}, M_{1}=50 \mathrm{GeV}$ and $\mu=130 \mathrm{GeV}$.
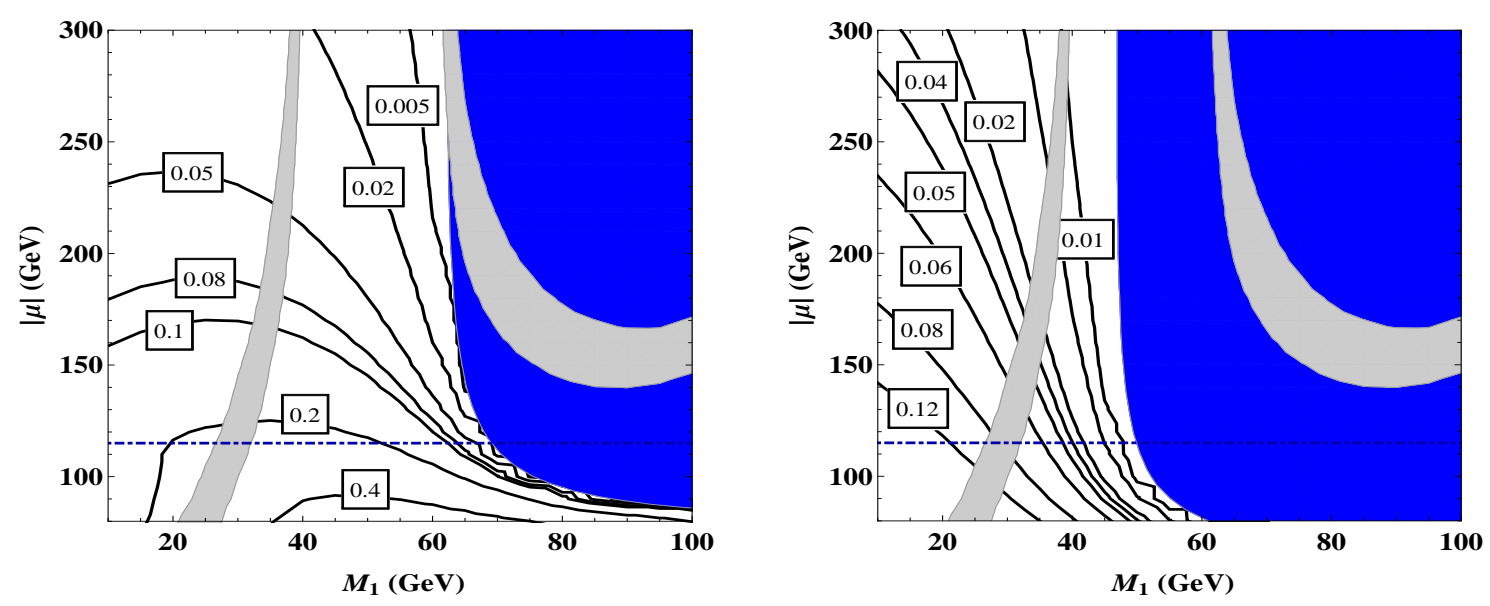

FIG. 3: The contours of constant branching ratio of $\left(H \rightarrow \tilde{\chi}_{1}^{0} \tilde{\chi}_{1}^{0}\right)$ (left) and $\left(h \rightarrow \tilde{\chi}_{1}^{0} \tilde{\chi}_{1}^{0}\right)$ (right) for a fixed value of tan $\beta$ $=6$ and $M_{2}=200 \mathrm{GeV}$ with $\mu<0$. The region shaded in Blue is the region kinematically not allowed, whereas the Grey shaded region is the one with relic density within the experimental limits.

The dependence of our result on the other input parameters is as follows. If the gaugino mass parameter $M_{2}$ is lowered, the mass bound of the chargino pushes up the Blue-DotDashed line in Fig. 2 to large values of $\mu$ and vice-versa. The coupling of the Higgs $(h / H)$ with neutralino decreases with the increase of $\tan \beta$ resulting in smaller invisible branching ratio. Large values of $\tan \beta$ as discussed before are disfavored in the non-decoupling scenario, by the LHC experiments. Since there is an enhancement in the branching ratio, if a smaller value of $\tan \beta$ is chosen, for the sake of completeness we also quote the results for $\tan \beta=4$. It can be seen from the left hand side of Fig. 2 that for $\tan \beta=6$, the largest possible branching ratio of $H$ to a pair of neutralinos is $8 \%$, if the LEP constraint on the chargino mass is considered. On the other hand if the value of $\tan \beta$ is reduced to 4 , the maximum possible BR satisfying the LEP constraint is $12 \%$. In case of $h$ the change is significant, as for $\tan \beta=6$, the maximum possible BR was around $28 \%$ as can be seen from the right hand side of Fig. 2 . For $\tan \beta=4$, this increases to about $40 \%$.

We next show in Fig. 3, the contours of the invisible branching ratio of CP even Higgs $H$ and $h$ to the lightest neutralinos for $\mu$ less than 0 . The values of the other parameters are same as before with $M_{2}=200 \mathrm{GeV}$ and $\tan \beta=6$. The chargino mass bound on $\mu$ decreases due to the increase in chargino mass. The branching ratio of $Z$ to a pair of neutralinos decreases, with negative $\mu$, therefore the considered $\mu-M_{1}$ parameter space is not constrained by Eq. (II.9) in this case. The neutralino mass also increases with negative $\mu$, resulting in larger regions of parameter space being kinematically not allowed. The invisible branching ratio of $H$ increases for negative $\mu$. This is mainly because for $\mu>0$, there is a cancellation between the terms $Z_{13} \cos \alpha$ and $Z_{14} \sin \alpha$ of (II.7), whereas these two terms add for $\mu<0$ leading to enhanced neutralino Higgs coupling. This behaviour is just the opposite for $h$ as can be seen from the right plot of Fig. 3. Here for $\mu<0$, there is a cancellation between the $Z_{13} \sin \alpha$ and $Z_{14} \cos \alpha$ terms of (II.6) leading to reduced coupling of Higgs to the neutralinos. Overall it can be seen from the above that in the non-decoupling regime it will not be possible to constrain 
the neutralino sector from the recent Higgs results unlike the decoupling regime where it was possible to do so [24]. More data from the LHC is needed so as to constrain the neutralino parameter space from the Higgs result in this case.

\section{DECAY OF THE HIGGS IN THE NMSSM}

We now extend the considerations of the previous section to the NMSSM which has a richer Higgs and neutralino sector. To recapitulate, the NMSSM has an extra gauge singlet superfield $S$ in addition to the two Higgs doublets $H_{1}$ and $H_{2}$, of the MSSM. The Higgs(ino) mass term $\mu H_{1} H_{2}$ in the superpotential of the MSSM is replaced by the trilinear coupling $\lambda \mathrm{SH}_{1} \mathrm{H}_{2}$, where $\lambda$ is a dimensionless coupling [35-41]. There is also an additional trilinear self coupling of the singlet $S^{3}$. The superpotential involving only the Higgs field then takes the form

$$
W_{N M S S M}=\lambda S H_{1} H_{2}-\frac{\kappa}{3} S^{3}
$$

Once the scalar potential of the singlet superfield acquires a vacuum expectation value $s$, the first term of the superpotential (III.1) then generates an effective $\mu$ term, where $\mu_{\text {eff }}=\lambda s$. This $\mu_{\text {eff }}$ term is naturally of the order of the electroweak scale, thereby providing a solution to the $\mu$ problem of MSSM. The Higgs sector of the NMSSM at tree level is described by six parameters $\mu_{e f f}, \lambda, \kappa, \tan \beta, A_{\lambda}$ and $A_{\kappa}$, compared to the Higgs sector of the MSSM, which is only defined by two independent parameters $\left(\tan \beta, M_{A}\right)$. The physical Higgs spectrum consists of 3 CP-even states, 2 CP-odd states along with a pair of charged Higgs boson. The neutralino sector in case of the NMSSM, due to the addition of the singlet becomes a $5 \times 5$ matrix, which can be written in the bino, wino, Higgsino and singlino basis [42-44]. It is described by six independent parameters $\mu_{e f f}, M_{1}, M_{2}, \tan \beta, \lambda$ and $\kappa$.

$$
M_{\mathrm{NMSSM}}=\left(\begin{array}{ccccc}
M_{1} & 0 & -M_{Z} \sin \theta_{W} \cos \beta & M_{Z} \sin \theta_{W} \sin \beta & 0 \\
0 & M_{2} & M_{Z} \cos \theta_{W} \cos \beta & -M_{Z} \cos \theta_{W} \sin \beta & 0 \\
-M_{Z} \sin \theta_{W} \cos \beta & M_{Z} \cos \theta_{W} \cos \beta & 0 & -\mu_{e f f} & -\lambda v_{2} \\
M_{Z} \sin \theta_{W} \sin \beta & -M_{Z} \cos \theta_{W} \sin \beta & -\mu_{e f f} & 0 & -\lambda v_{1} \\
0 & 0 & -\lambda v_{2} & -\lambda v_{1} & 2 \kappa x
\end{array}\right) .
$$

For a massless neutralino the determinant of the mass matrix (III.2) should be zero, which leads to [15]

$$
2 \kappa x \mu_{e f f}\left(\Delta_{0} \sin 2 \beta-\mu_{e f f} M_{1} M_{2}\right)+\lambda^{2} v^{2}\left[\Delta_{0}-\mu_{e f f} M_{1} M_{2} \sin 2 \beta\right]=0,
$$

where $\Delta_{0}=M_{Z}^{2}\left(M_{1} \cos ^{2} \theta_{W}+M_{2} \sin ^{2} \theta_{W}\right)$. (III.3) in turn leads to the following condition

$$
\kappa=\frac{\lambda}{2}\left(\frac{\lambda v}{\mu_{e f f}}\right)^{2} \frac{\Delta_{0}-\mu_{e f f} M_{1} M_{2} \sin 2 \beta}{\mu_{e f f} M_{1} M_{2}-\Delta_{0} \sin 2 \beta},
$$

for a massless neutralino in the NMSSM.

Analogous to the MSSM, even in case of the NMSSM it was earlier investigated, whether the recent global fits from the Higgs data can constrain the parameter space of the neutralino sector [13], with the lightest Higgs $\left(h_{1}\right)$ of the NMSSM, being identified as the $126 \mathrm{GeV}$ state observed at LHC. There can also be another possibility where the second lightest CP even Higgs $\left(h_{2}\right)$ will lead to a SM like Higgs boson in the mass range $124 \mathrm{GeV}$ $\leq M_{h_{2}} \leq 127 \mathrm{GeV}$. The mass of the lightest CP even Higgs $h_{1}$ and sometimes the lightest pseudoscalar Higgs $a_{1}$ will be less than $M_{h_{2}}$, and in some regions of the parameter space, the decay of $h_{2}$ to a pair of $h_{1}$ or $a_{1}$ will be kinematically allowed. A lot of work has been done in the context of two light Higgs boson within NMSSM. Various scenarios have been proposed in this context and are examined or constrained in the light of the recent LHC results.

(a) One of them was proposed to explain the enhancement of the Higgs signal in some of the channels relative to the SM. The authors of [45-47] have identified a set of parameter space, in the context of NMSSM where the two lightest CP even Higgs boson are found to be closely degenerate and lie in the mass window 123-128 GeV. We do not consider this possibility here.

(b) Another scenario that has been widely considered in the context of NMSSM, is where the heavier Higgs boson $h_{2}$ is considered as the SM like Higgs in the mass range of [124, 127] GeV and the lighter Higgs boson $h_{1}$ is around $98 \mathrm{GeV}$ in order to account for the LEP excess [48]. We will refer to this as the $98+$ $126 \mathrm{GeV}$ Higgs scenario further in the text. 


\begin{tabular}{||c|c|c||}
\hline parameter & lower range & upper range \\
\hline \hline$\mu_{e f f}$ & 100 & 400 \\
$\tan \beta$ & 5 & 40 \\
$\lambda$ & 0.01 & 0.7 \\
$\kappa$ & 0.01 & 0.6 \\
$A_{\lambda}$ & -500 & 1000 \\
$A_{\kappa}$ & -1000 & 100 \\
\hline
\end{tabular}

TABLE II: Ranges of the input parameters of the NMSSM of our scan

Since $h_{1}$ is in the mass range $(96-100) \mathrm{GeV}$, in order to respect the LEP limit of $C_{e f f}^{2 b}=\left[g_{Z Z h}^{2} / g_{Z Z h_{S M}}^{2}\right] \mathrm{BR}(h \rightarrow$ $b \bar{b}$ ), from the process $e^{+} e^{-} \rightarrow h Z \rightarrow b \bar{b} Z$, the mass of the lightest pseudoscalar $a_{1}$ is assumed to be less than $2 M_{b}$. There has been additional constraints on the mass of $a_{1}$ from various other experiments. In a recent result from CMS, the experiment has excluded pseudoscalar mass in the range $1 \mathrm{GeV}<M_{a_{1}}<2 M_{\tau}$, for a scalar Higgs in the mass range $86-150 \mathrm{GeV}$. Therefore in order to study the $98+126 \mathrm{GeV}$ scenario, the light pseudoscalar should be either in the range $2 M_{\tau}<M_{a_{1}}<2 M_{b}$ or heavier than $M_{h_{2}}$. Another way of evading the CMS bound in this two light Higgs scenario, is to consider the mass of the lightest scalar $\left(M_{h_{1}}\right)$ to be less than $86 \mathrm{GeV}$ [49]. The LEP searches of a Higgs boson decaying into four $\tau$ leptons via intermediate pseudoscalar [50], has placed constraint on the combined production times branching ratio on the $4 \tau$ 's decay channel $\left.\left(\sigma\left(e^{+} e^{-} \rightarrow Z h\right) / \sigma_{S M}\left(e^{+} e^{-} \rightarrow Z h\right)\right) \times \mathrm{BR}\left(h \rightarrow a_{1} a_{1}\right) \times \mathrm{BR}\left(a_{1} \rightarrow \tau^{+} \tau^{-}\right)^{2}<1\right)$. All these searches have mainly considered the decay of Higgs to pseudoscalar as the only non-standard decay mode apart from the usual SM decay channels. Since in our analyses, there are other non-standard decay modes of the Higgs boson, like the Higgs decaying to a pair of lightest neutralinos, the constraint on the mass of the pseudoscalar will be lightened by the presence of these additional decay channels. We have considered the mass of the pseudoscalar such that the LEP limit from the process $e^{+} e^{-} \rightarrow h Z, h \rightarrow b \bar{b}$ is satisfied along with the process $e^{+} e^{-} \rightarrow h Z, h \rightarrow a_{1} a_{1}, a_{1} \rightarrow b \bar{b}$. In addition we have also seen that the LEP constraint on the $4 \tau$ 's final state is also satisfied.

The Higgs sector of the NMSSM being described by six independent parameters, $\mu_{e f f}, \lambda, \kappa, \tan \beta, A_{\lambda}$ and $A_{\kappa}$, a scan is performed over a million random points in the range of parameters listed in Table II. We have used NMSSMTools-4.1.0 [51,52] for our analysis. The scan includes all the recent experimental constraints from the Higgs, flavour and precision electroweak measurements implemented within NMSSMTools. We have additionally demanded that the second lightest CP even Higgs of the NMSSM $\left(h_{2}\right)$ should lead to a SM like Higgs in the mass range $[124,127] \mathrm{GeV}$. We have also restricted to values of $\kappa$ and $\lambda$ less than 0.7 . This is due to the theoretical constraint that there should be no charge and color breaking global minima of the scalar potential and that a Landau pole does not develop below the GUT scale. Since the Higgs mass spectrum is independent of the gaugino mass parameters, we have considered universal boundary conditions at the GUT scale, with the $S U(3)_{C}$ gaugino mass parameter $M_{3}=1400 \mathrm{GeV}$, from the gluino searches at the LHC. The remaining two soft SUSY breaking gaugino parameters have values $M_{1}=197 \mathrm{GeV}$ and $M_{2}=395 \mathrm{GeV}$. In this work, we have divided the points which survive all the constraints defined above into two distinct scenarios.

- Scenario 1: The heavier Higgs boson $h_{2}$ is in the mass range $[124,127] \mathrm{GeV}$, whereas the lightest pseudoscalar $a_{1}$ has a mass less than half the mass of $h_{2}$. The lightest CP even Higgs $h_{1}$ is lighter than $h_{2}$.

- Scenario 2: As before $h_{2}$ in the mass range $[124,127] \mathrm{GeV}$, with the lightest CP even scalar $h_{1}$ less than half the mass of $h_{2}$. The lightest pseudoscalar $a_{1}$ can be lighter than $h_{1}$ satisfying the experimental constraints or heavier than $h_{2}$.

The $98+126 \mathrm{GeV}$ Higgs Boson case, can be obtained in the first scenario, but we separately give the parameter points which satisfy this. We show in Figs. 4, 5, 6 the different parameters, that lead to the scenarios of interest considered here. The points in the plots as discussed before satisfies all the experimental constraints. It can be seen from Fig. 4 that smaller values of $\lambda$ and $\kappa$ are preferred for the scenarios we are considering. A lot of work has been done in the context of large doublet singlet mixing in the Higgs sector, i.e. concentrating on regions of the parameter space with large values of $\lambda$, leading naturally to a SM like Higgs $h_{2}$ in the 126 $\mathrm{GeV}$ range [53-56]. But we are mainly interested in the case where the Higgs decay channels to non-standard particles are open, such as $h_{2} \rightarrow a_{1} a_{1}, Z a_{1}, h_{1} h_{1}$, along with the neutralinos. Most of the points which satisfy the above constraints are concentrated in the low $\kappa-\lambda$ plane, therefore we show them here. Moreover Scenario 1 is distinct from the others in the $A_{\lambda}-A_{\kappa}$ plane, since $M_{a_{1}}$ is sensitive to $A_{\kappa}$. With the universal gaugino masses at the GUT scale, and from (III.4) we find that it is not possible to get a massless neutralino in the 


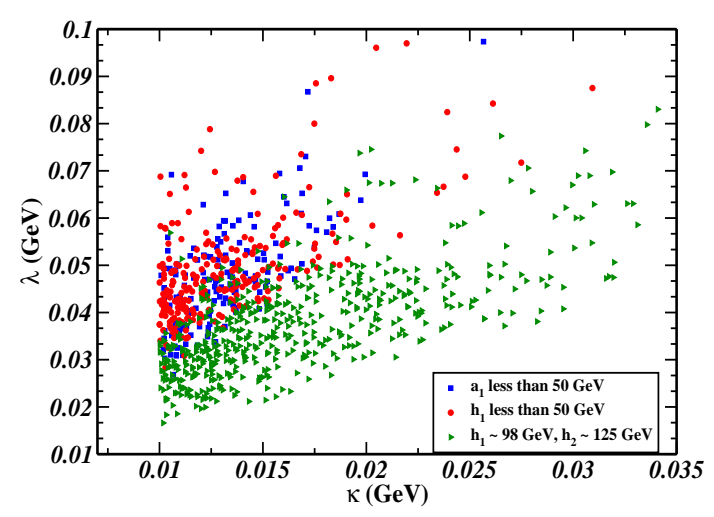

FIG. 4: Point satisfying all the experimental constraints in the $\lambda-\kappa$ plane.

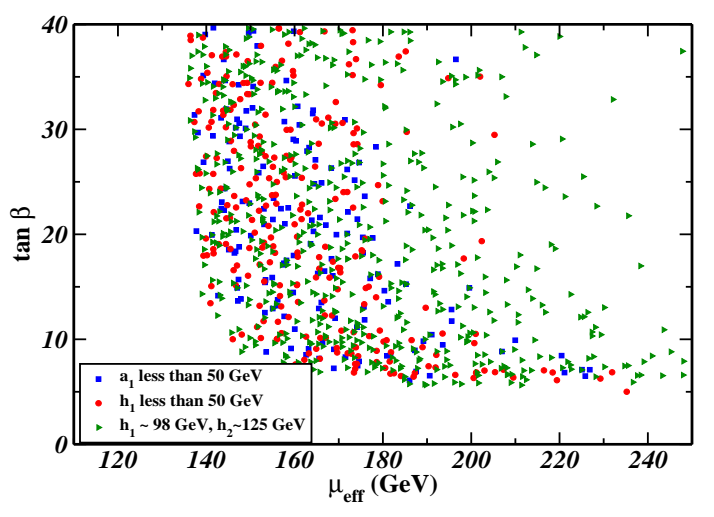

FIG. 5: Point satisfying all the experimental constraints in the $\tan \beta-\mu_{\text {eff }}$ plane.

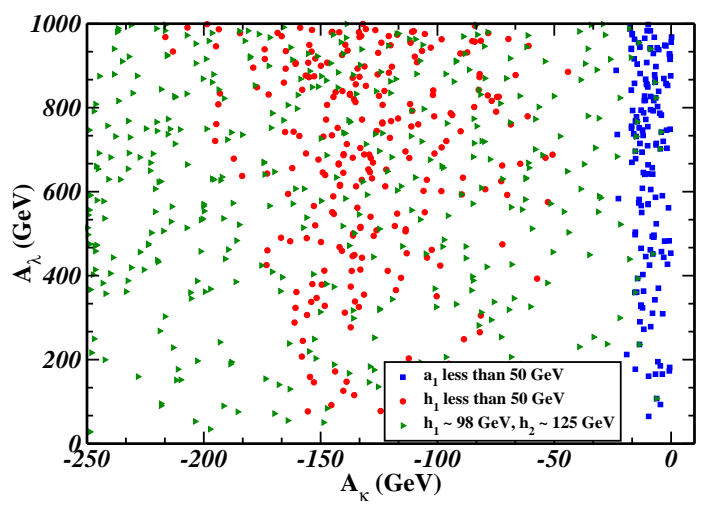

FIG. 6: Point satisfying all the experimental constraints in the $A_{\lambda}-A_{\kappa}$ plane.

\begin{tabular}{||c|c|c|c|c|c|c|c||}
\hline & $\lambda$ & $\kappa$ & $A_{\lambda}$ & $A_{\kappa}$ & $\tan \beta$ & $\mu_{e f f}$ & $A_{t, b, \tau}$ \\
\hline \hline Scenario 1 & 0.055 & 0.013 & 875.76 & -0.174 & 19.97 & 169.47 & -2500 \\
Scenario 2 & 0.037 & 0.013 & 978.21 & -168.44 & 18.69 & 149.77 & -2500 \\
\hline
\end{tabular}

TABLE III: Input parameters for the Benchmark Points in case of NMSSM

NMSSM, with $M_{h_{2}} \approx 126 \mathrm{GeV}$. This result holds in the entire parameter space considered in our analyses. In the range of the parameter space considered by us, for NMSSM with universal boundary conditions of gaugino masses at the GUT scale, the decay $h_{2} \rightarrow \tilde{\chi}_{1}^{0} \tilde{\chi}_{1}^{0}$ is kinematically possible in some regions. Since analysis with the GUT relation between $M_{1}$ and $M_{2}$ will result in confining to a particular case, we do not consider that possibility here but instead concentrate on the general case with $M_{1}$ and $M_{2}$ as independent parameters. We consider a benchmark point for the different scenarios listed above and present the results here. The spectrum of the sparticles and the gluinos are considered similar to the case of MSSM. We list in the Table III the parameters for the two different benchmark scenarios considered here. The corresponding Higgs spectrum is listed in Table IV.

We show in Fig. 7, the contours of constant branching ratios of $h_{2} \rightarrow \tilde{\chi}_{1}^{0} \tilde{\chi}_{1}^{0}$ (Black) and $h_{2} \rightarrow \tilde{\chi}_{1}^{0} \tilde{\chi}_{2}^{0}($ RedDashed) in the $\mu_{\text {eff }}-M_{1}$ plane with the values of the other parameters fixed as given in Table III for Scenario 1. Since $M_{h_{2}}$ is sensitive to $\mu_{e f f}$, we have varied $\mu_{e f f}$ in the range such that $M_{h_{2}} \approx 124-127 \mathrm{GeV}$. The blue shaded region is the area where the $h_{2}$ decay to the lightest neutralinos is kinematically not accessible. The lightest neutralino has a dominant gaugino component, in the entire $\mu_{e f f}-M_{1}$ plane. The singlino component is absent for low $M_{1}$ values, and open up at higher values of $M_{1}$. The grey shaded area shows the region where 


\begin{tabular}{||c|c|c|c|c|c|c||}
\hline & $M_{h_{1}}$ & $M_{h_{2}}$ & $M_{h_{3}}$ & $M_{a_{1}}$ & $M_{a_{2}}$ & $M_{h^{ \pm}}$ \\
\hline \hline Scenario 1 & 76.28 & 126.47 & 1716.62 & 5.23 & 1716.6 & 1718.2 \\
Scenario 2 & 47.69 & 124.58 & 1657.66 & 164.07 & 1657.63 & 1659.38 \\
\hline
\end{tabular}

TABLE IV: The Higgs mass spectrum for the different Benchmark Scenarios in case of the NMSSM

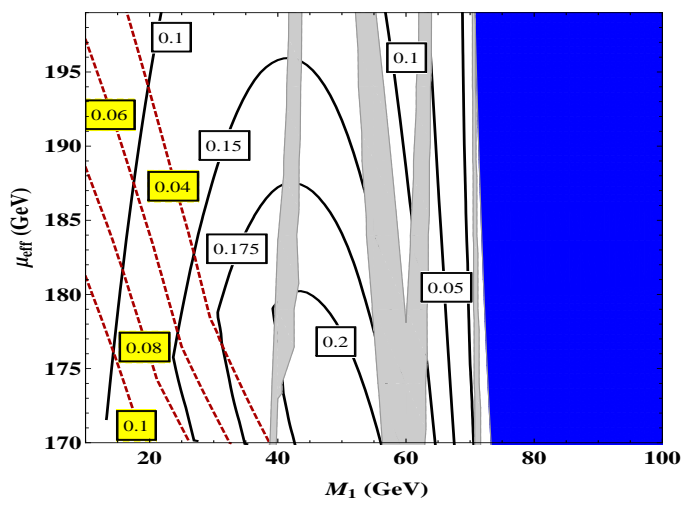

FIG. 7: Contours of constant branching ratio of $\left(h_{2} \rightarrow\right.$ $\left.\tilde{\chi}_{1}^{0} \tilde{\chi}_{1}^{0}\right)$ [Black-Solid] and $\left(h_{2} \rightarrow \tilde{\chi}_{1}^{0} \tilde{\chi}_{2}^{0}\right)$ [Red-Dashed] in NMSSM in the $\mu_{\text {eff }}-M_{1}$ plane for a fixed value of $M_{2}$ $=200 \mathrm{GeV}$ and the other parameters fixed to values in Table III for Scenario 1.

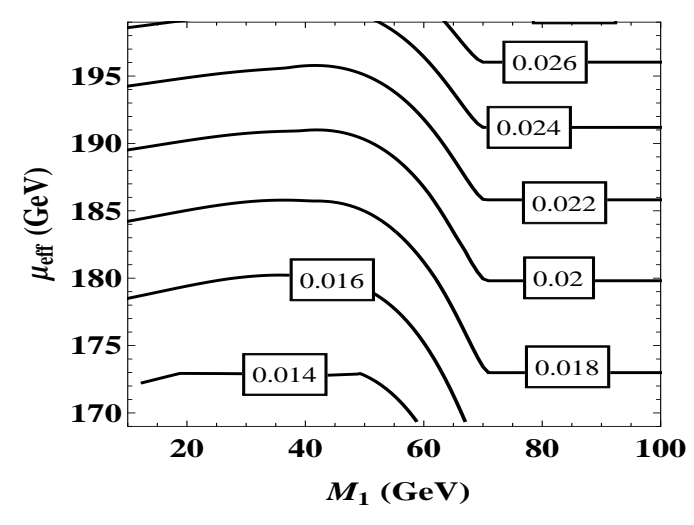

FIG. 8: Contours of constant branching ratio of $\left(h_{2} \rightarrow\right.$ $\left.a_{1} a_{1}\right)$ in NMSSM in the $\mu_{e f f}-M_{1}$ plane for a fixed value of $M_{2}=200 \mathrm{GeV}$ and the other parameters fixed to values in Table III for Scenario 1.

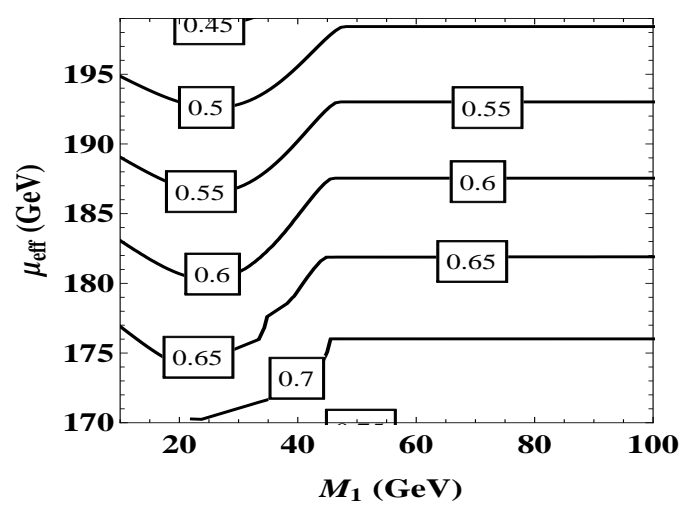

FIG. 9: Contours of constant branching ratio of $\left(h_{1} \rightarrow\right.$ $\left.a_{1} a_{1}\right)$ in NMSSM in the $\mu_{e f f}-M_{1}$ plane for a fixed value of $M_{2}=200 \mathrm{GeV}$ and the other parameters fixed to values in Table III for Scenario 1.

the lightest neutralino satisfies the relic density constraint. The relic density is satisfied in the region, where the neutralino is a gaugino-higgsino mixture, but has a dominant gaugino component. We see that for the region allowed by the relic density, the invisible branching ratio can vary between the range of $15 \%-20 \%$. As invisible BRs less that $38 \%$ is still allowed by the global fits, the NMSSM parameter space can not be constrained by the present LHC Higgs data. In the future with the upgraded LHC results, it will be possible to constrain the parameter space from the Higgs data. The nature of the contours in Fig. 7 can be readily understood from the fact that, since $M_{\tilde{\chi}_{2}^{0}}$ also depends on $\mu_{e f f}$ and $M_{1}$, at low values of $M_{1}$, the decay channel $h_{2} \rightarrow \tilde{\chi}_{1}^{0} \tilde{\chi}_{2}^{0}$ is kinematically accessible. This is shown by Red-Dashed lines which decreases with increasing $M_{1}$ due to the increase in mass of $\tilde{\chi}_{2}^{0}$, and the opening of the channel $h_{2} \rightarrow \tilde{\chi}_{1}^{0} \tilde{\chi}_{1}^{0}$. The second lightest neutralino is mostly a singlino. Since in this scenario the lightest pseudoscalar is very light, $M_{a_{1}} \approx 6 \mathrm{GeV}$, we show in Fig. 8 , the branching ratio of $h_{2}$ to a pair of $a_{1}$ in the $\mu_{e f f}-M_{1}$ plane. The decay channel $h_{2} \rightarrow Z a_{1}$ is also open. but 
the branching ratio is very small. So we do not consider it here. The nature of the contour in Fig. 8 can be understood from the fact that at high values of $M_{1}$ since the neutralino decay channel is not there, the BR is constant. Whereas for lower values of $M_{1}$ due to the invisible branching ratio, the contours show a curved nature. Here $a_{1}$ will mostly decay into a pair of $\tau$ 's, which can be easily detected in the collider. So we do not include them in the calculation of the invisible branching ratio. We also show in Fig. 9 the decay of the light CP even Higgs $h_{1}$ to a pair of $a_{1}$. Here $h_{1}$ predominantly decays to $a_{1}$ with around 50-60\% branching ratio. The dip in the contours at lower values of $M_{1}$ is due to the presence of light neutralinos, leading to the decay channel $h_{1} \rightarrow \tilde{\chi}_{1}^{0} \tilde{\chi}_{1}^{0}$. The lighter Higgs $h_{1}$ in this case can be observed in the collider through the decay mode $h_{1} \rightarrow a_{1} a_{1} \rightarrow 4 \tau$ 's. We next show our results for Scenario 2 , where $M_{h_{1}} \leq M_{h_{2}} / 2$. The nature of the

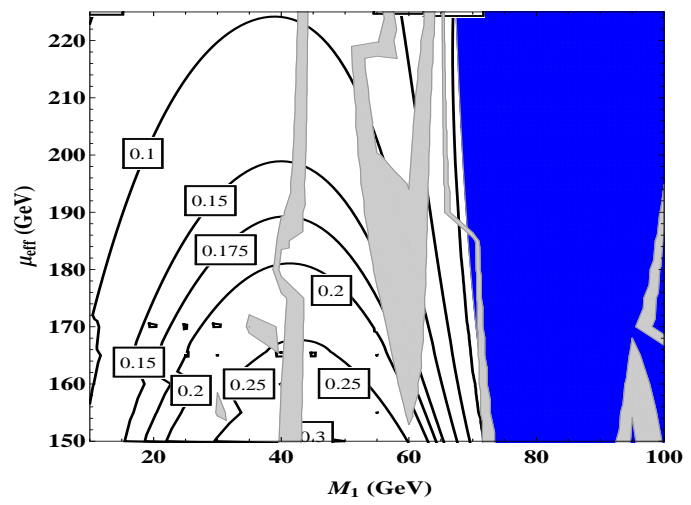

FIG. 10: Contours of constant branching ratio of $\left(h_{2} \rightarrow \tilde{\chi}_{1}^{0} \tilde{\chi}_{1}^{0}\right)$ in NMSSM in the $\mu_{e f f}-M_{1}$ plane for a fixed value of $M_{2}=200 \mathrm{GeV}$ and the other parameters fixed to values in Table III for Scenario 2 .

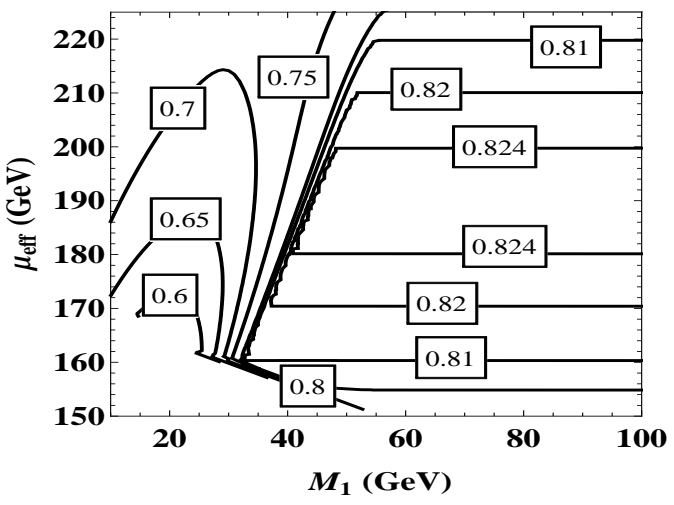

FIG. 11: Contours of constant branching ratio of $\left(h_{1} \rightarrow b \bar{b}\right)$ in NMSSM in the $\mu_{e f f}-M_{1}$ plane for a fixed value of $M_{2}=200 \mathrm{GeV}$ and the other parameters fixed to values in Table III for Scenario 2 .

Fig. 10 is similar to Fig. 7 showing the contours of constant branching ratios of $h_{2} \rightarrow \tilde{\chi}_{1}^{0} \tilde{\chi}_{1}^{0}$. The value of $\lambda$ in Scenario 2 is smaller compared to that of Scenario 1, as can be seen from Table III. Since the neutralino mass $\left(M_{\tilde{\chi}_{1,2}^{0}}\right)$ increases with decreasing $\lambda$, the neutralinos in this case are more massive. Therefore the decay channel $h_{2} \rightarrow \tilde{\chi}_{1}^{0} \tilde{\chi}_{2}^{0}$ is kinematically not accessible. The allowed parameter space can only be constrained by the future LHC results. We would further add that the composition of the lightest and the second lightest neutralino is similar to Scenario 1. Finally we show in Fig. 11, the contours of constant branching ratio of the lightest CP even Higgs to a pair of $b$ quarks, which is the dominant decay mode. The BR can be as high as $80 \%$ for most of the $\mu_{\text {eff }}-M_{1}$ parameter space. When the neutralino is light enough allowing for the invisible decay mode of the light Higgs $\left(h_{1}\right)$, the BR decrease by about $20 \%$. It will be possible to observe this state at the LHC, through the $b \bar{b}$ decay mode. The lightest pseudoscalar Higgs in this scenario is heavier than $h_{1}$ and $h_{2}$, but is considerably lighter to be observed at the LHC. Nevertheless the dominant decay mode of $a$ will be to a pair of neutralinos, and the second dominant mode will be to a pair of $b$ quarks. The pseudoscalar $a$ will therefore be difficult to be observed in the LHC, due to a large invisible BR. This shows that the global fits from the recent LHC data is unable to constrain the neutralino sector of the NMSSM, when the second lightest scalar is identified as the $126 \mathrm{GeV}$ Higgs. We would like to add that the analysis carried out in this work can also be repeated for negative values of $\kappa$. However, we have found that in this case for most of the parameter space with $h_{2}$ around $126 \mathrm{GeV}$, the limits on the invisible branching ratio cannot constrain the parameter space as is the case with positive values of $\kappa$. A more detailed precision study in the Higgs sector is allowed so as to constrain the parameter space in this case.

\section{CONCLUSION}

In this work we have considered the possibility of the invisible decays of the second lightest CP even Higgs boson in the context of both MSSM and NMSSM. The second lightest Higgs behaves as the SM like Higgs with mass in the allowed mass range $\approx[124,127] \mathrm{GeV}$. The neutralino sector in the last few years has been studied in details and also constrained by the data from different astrophysical, cosmological and collider experiments. The recent LHC results on the Higgs branching ratio has independently constrained the Higgs invisible branching ratio, through global fits. The Higgs can decay to a pair of neutralinos giving rise to invisible 
decay branching ratio provided $M_{\tilde{\chi}_{1}^{0}}<M_{H}$. This invisible Higgs decay channel is also looked through direct searches at the LHC. It will therefore be important to consider the implications of the current information on Higgs from the LHC, on the neutralino sector of the supersymmetric models. In the context of MSSM, we discuss in brief whether it will be possible for the SM like Higgs to decay to a pair of neutralinos, with both universal and nonuniversal gaugino masses at the GUT scale. We find that with universal gaugino masses, the invisible decay channel $H \rightarrow \tilde{\chi}_{1}^{0} \tilde{\chi}_{1}^{0}$ is kinematically not allowed. Nevertheless in case of nonuniversal gaugino masses, for certain representations of $S O(10)$ and $E_{6}$, the $126 \mathrm{GeV}$ Higgs will have a considerable invisible branching ratio. We have then analyzed the possibility of having a large invisible branching ratio in the context of NMSSM which has a richer neutralino and Higgs sector compared to the MSSM. We find that in case of the NMSSM it is possible to have the invisible decay channel $\left(h_{2} \rightarrow \tilde{\chi}_{1}^{0} \tilde{\chi}_{1}^{0}\right)$ with universal gaugino masses at the GUT scale. The assumption of the GUT relation between $M_{1}$ and $M_{2}$ being largely model dependent, we have considered a more general case and have done our analyses by treating $M_{1}$ and $M_{2}$ as two independent parameters in case of both the MSSM and the NMSSM. With this assumption, there is an additional freedom of the neutralino being very light.

We have then studied the decay of the Higgs bosons ( $h$ and $H$ ) to a pair of lightest neutralinos in the context of the non-decoupling scenario of the MSSM. We have considered the possibility that the $126 \mathrm{GeV}$ scalar $(H)$ observed at the LHC along with the $98 \mathrm{GeV}$ scalar $(h)$ from the LEP excess in the $b \bar{b}$ final state can be concurrently explained in the MSSM framework. We find that there are regions in the MSSM parameter space where such scenarios exist. The neutralino sector being dependent on the parameters $\mu, M_{1}, M_{2}, \tan \beta$, we give our results for a fixed value of $\tan \beta$ and $M_{2}$ in the $\mu-M_{1}$ plane because we are mainly interested in the Higgs decay channel to a pair of neutralinos. We find the invisible BR in this case is too small to be constrained by the recent LHC fits from the Higgs data. This is in contrast to the case when the lightest CP even Higgs boson $(h)$ was SM like $[13,24]$, the decoupling scenario. There it was found that with $h \approx 126$ $\mathrm{GeV}$, a large portion of the $\mu-M_{1}$ parameter space for a fixed value of $\tan \beta$ and $M_{2}$ allowed a large invisible branching ratio in conflict with the latest LHC fits from the Higgs sector, thereby constraining the neutralino parameter space. Therefore if the non-decoupling scenario exists, the parameter space of the neutralino sector cannot be constrained by the recent LHC data. Higher precision Higgs physics is required to constrain the parameter space in the scenario. This scenario can be alternatively tested by looking for the other Higgs bosons production, $M_{h} \approx 98 \mathrm{GeV}, M_{A} \approx 100-150 \mathrm{GeV}$ and $M_{H^{ \pm}} \approx 150-200 \mathrm{GeV}$ at the LHC. We do not consider the possibility here. The dependence of our results on the other input parameters is also discussed in details.

An analogous analysis is then performed in the context of the NMSSM. The number of independent parameters in the neutralino and the Higgs sector is greater than that of the MSSM. We have performed a scan over the parameters contributing to the neutralino sector of the NMSSM and have plotted the points which pass the various theoretical and experimental constraints discussed in the text, along with the condition that the second lightest Higgs $h_{2}$ behaves like the SM Higgs boson. The points which survive these constraints are then divided into three scenarios, depending on the mass of the other Higgs bosons. We firstly separately isolate the points where both $M_{h_{1}, a_{1}}$ is less than $m_{h_{2}}$, but only the decay of $h_{2}$ to a pair of CP odd Higgs $a_{1}$ is kinematically allowed. Secondly we consider the case when the mass of the lightest Higgs boson $\left(h_{1}\right)$ is less than half the mass of $h_{2}$, that is the decay $h_{2} \rightarrow h_{1} h_{1}$ is kinematically allowed. We have considered a single benchmark point for these two scenarios and have exclusively worked out the results. The dependence of the results on the other points can be suitably interpreted. There are regions in the parameter space which also satisfies the $98\left(h_{1}\right)+$ $126\left(h_{2}\right) \mathrm{GeV}$ Higgs scenario, so as to account for the LEP excess along with the Higgs data from the LHC. We list the points which fall in this parameter space, but we do not consider benchmark point in this case as the results will be similar to the first scenario. We see that the invisible branching ratio reaches a maximum of $20 \%$, for the two scenarios considered here, still too small to be be constrained by the recent LHC fits from the Higgs data. The result is thus similar to MSSM, where with the second lightest scalar being $126 \mathrm{GeV}$, it is currently not possible to constrain the neutralino parameter space from the Higgs data. The situation will improve with more data from the next LHC run.

Overall we find that in the context of both MSSM and NMSSM, it is not possible to constrain the neutralino sector from the recent Higgs data, if the second lightest scalar is identified with the one observed at the LHC. The presence of the other light Higgs will however lead to interesting collider signals at the LHC, which will alternatively test these scenarios. It will be worthwhile to do a detailed collider study of these light Higgs and find the reach of the LHC. The direct detection of some susy particles in the 13 and $14 \mathrm{TeV}$ runs of the LHC would significantly cut down the arbitrariness of extensions of the SM to its susy variants after which a precision Higgs era could be pursued at the LHC. Hopefully this can be pursued in the near future. 


\section{ACKNOWLEDGEMENTS}

We would like to thank B. Ananthanarayan for a careful reading of the manuscript and constructive suggestions. MP would also like to thank Dipan Sengupta for help regarding micrOMEGAs. The work of PNP is supported by the J. C. Bose National Fellowship of the Department of Science and Technology, India, and by the Council of Scientific and Industrial Research, India.

[1] G. Aad et al. [ATLAS Collaboration], Phys. Rev. D 86, 032003 (2012) [arXiv:1207.0319 [hep-ex]].

[2] S. Chatrchyan et al. [CMS Collaboration], JHEP 1306, 081 (2013) [arXiv:1303.4571 [hep-ex]].

[3] L. Calibbi, J. M. Lindert, T. Ota and Y. Takanishi, JHEP 1310, 132 (2013) [arXiv:1307.4119].

[4] J. Kozaczuk and S. Profumo, arXiv:1308.5705 [hep-ph].

[5] A. Arbey, M. Battaglia and F. Mahmoudi, Phys. Rev. D 88, 095001 (2013) [arXiv:1308.2153 [hep-ph]].

[6] K. Hagiwara, S. Mukhopadhyay and J. Nakamura, Phys. Rev. D 89, 015023 (2014) [arXiv:1308.6738 [hep-ph]].

[7] G. Belanger, B. Dumont, U. Ellwanger, J. F. Gunion and S. Kraml, Phys. Rev. D 88, 075008 (2013) [arXiv:1306.2941 [hep-ph]].

[8] A. Djouadi and G. g. Moreau, arXiv:1303.6591 [hep-ph].

[9] G. Aad et al. [ATLAS Collaboration], arXiv:1402.3244 [hep-ex].

[10] [ATLAS Collaboration], ATLAS-CONF-2013-011.

[11] [CMS Collaboration], "Search for invisible Higgs produced in association with a Z boson," CMS-PAS-HIG-13-018.

[12] [CMS Collaboration], "Search for invisible Higgs decays in the VBF channel," CMS-PAS-HIG-13-013.

[13] B. Ananthanarayan, J. Lahiri, P. N. Pandita and M. Patra, Phys. Rev. D 87, 115021 (2013) [arXiv:1306.1291 [hep-ph]].

[14] R. Barate et al. [LEP Working Group for Higgs boson searches and ALEPH and DELPHI and L3 and OPAL Collaborations], Phys. Lett. B 565, 61 (2003) [hep-ex/0306033].

[15] I. Gogoladze, J. D. Lykken, C. Macesanu and S. Nandi, Phys. Rev. D 68, 073004 (2003) [hep-ph/0211391].

[16] S. Schael et al. [ALEPH and DELPHI and L3 and OPAL and LEP Working Group for Higgs Boson Searches Collaborations], Eur. Phys. J. C 47, 547 (2006) [hep-ex/0602042].

[17] S. Chatrchyan et al. [CMS Collaboration], Phys. Lett. B 713, 68 (2012) [arXiv:1202.4083 [hep-ex]].

[18] G. Aad et al. [ATLAS Collaboration], JHEP 1302, 095 (2013) [arXiv:1211.6956 [hep-ex]].

[19] [CMS Collaboration], CMS-PAS-HIG-12-050.

[20] [ATLAS Collaboration], ATLAS-CONF-2012-160.

[21] E. Boos, A. Djouadi, M. Muhlleitner and A. Vologdin, Phys. Rev. D 66, 055004 (2002) [hep-ph/0205160].

[22] E. Boos, A. Djouadi and A. Nikitenko, Phys. Lett. B 578, 384 (2004) [hep-ph/0307079].

[23] N. D. Christensen, T. Han and S. Su, Phys. Rev. D 85, 115018 (2012) [arXiv:1203.3207 [hep-ph]].

[24] H. K. Dreiner, J. S. Kim and O. Lebedev, Phys. Lett. B 715, 199 (2012) [arXiv:1206.3096 [hep-ph]].

[25] A. Bartl, H. Fraas, W. Majerotto and N. Oshimo, Phys. Rev. D 40, 1594 (1989).

[26] H. E. Haber and G. L. Kane, Phys. Rept. 117, 75 (1985).

[27] J. Abdallah et al. [DELPHI Collaboration], Eur. Phys. J. C 31, 421 (2003) [hep-ex/0311019].

[28] K. Griest and H. E. Haber, Phys. Rev. D 37, 719 (1988).

[29] S. Heinemeyer, W. Hollik, A. M. Weber and G. Weiglein, JHEP 0804, 039 (2008) [arXiv:0710.2972 [hep-ph]].

[30] S. Schael et al. [ALEPH and DELPHI and L3 and OPAL and SLD and LEP Electroweak Working Group and SLD Electroweak Group and SLD Heavy Flavour Group Collaborations], Phys. Rept. 427, 257 (2006) [hep-ex/0509008].

[31] C. Bennett et al. [WMAP Collaboration], Astrophys. J. Suppl. 148, 97 (2003) [astro-ph/0302208].

[32] D. N. Spergel et al. [WMAP Collaboration], Astrophys. J. Suppl. 148, 175 (2003) [astro-ph/0302209].

[33] G. Belanger, F. Boudjema, A. Pukhov and A. Semenov, Comput. Phys. Commun. 174, 577 (2006) [hep-ph/0405253]. G. Belanger, F. Boudjema, A. Pukhov and A. Semenov, Comput. Phys. Commun. 149, 103 (2002) [hep-ph/0112278]. G. Belanger, F. Boudjema, A. Pukhov and A. Semenov, Comput. Phys. Commun. 185, 960 (2014) [arXiv:1305.0237 [hep-ph]].

[34] A. Belyaev, N. D. Christensen and A. Pukhov, Comput. Phys. Commun. 184, 1729 (2013) [arXiv:1207.6082 [hep$\mathrm{ph}]]$.

[35] P. Fayet, Nucl. Phys. B 90, 104 (1975).

H. P. Nilles, M. Srednicki and D. Wyler, Phys. Lett. B 120, 346 (1983).

[36] J. R. Ellis, J. F. Gunion, H. E. Haber, L. Roszkowski and F. Zwirner, Phys. Rev. D 39, 844 (1989).

[37] M. Drees, Int. J. Mod. Phys. A 4, 3635 (1989).

[38] P. N. Pandita, Phys. Lett. B 318, 338 (1993).

[39] P. N. Pandita, Z. Phys. C 59, 575 (1993).

[40] U. Ellwanger, Phys. Lett. B 303, 271 (1993) [hep-ph/9302224].

[41] T. Elliott, S. F. King and P. L. White, Phys. Lett. B 314, 56 (1993) [hep-ph/9305282].

[42] P. N. Pandita, Phys. Rev. D 50, 571 (1994).

[43] P. N. Pandita, Z. Phys. C 63, 659 (1994).

[44] S. Y. Choi, D. J. Miller, 2 and P. M. Zerwas, Nucl. Phys. B 711, 83 (2005) [hep-ph/0407209]. 
[45] G. Belanger, U. Ellwanger, J. F. Gunion, Y. Jiang and S. Kraml, arXiv:1208.4952 [hep-ph].

[46] J. F. Gunion, Y. Jiang and S. Kraml, Phys. Rev. Lett. 110, 051801 (2013) [arXiv:1208.1817 [hep-ph]].

[47] J. F. Gunion, Y. Jiang and S. Kraml, Phys. Rev. D 86, 071702 (2012) [arXiv:1207.1545 [hep-ph]].

[48] D. G. Cerdeno, P. Ghosh and C. B. Park, JHEP 1306, 031 (2013) [arXiv:1301.1325 [hep-ph]].

[49] S. Chatrchyan et al. [CMS Collaboration], Phys. Lett. B 726, 564 (2013) [arXiv:1210.7619 [hep-ex]].

[50] S. Schael et al. [ALEPH Collaboration], JHEP 1005, 049 (2010) [arXiv:1003.0705 [hep-ex]].

[51] http://www.th.u-psud.fr/NMHDECAY/nmssmtools.html

[52] U. Ellwanger, J. F. Gunion and C. Hugonie, JHEP 0502, 066 (2005) [hep-ph/0406215].

U. Ellwanger and C. Hugonie, Comput. Phys. Commun. 175, 290 (2006) [hep-ph/0508022].

[53] U. Ellwanger, JHEP 1203, 044 (2012) [arXiv:1112.3548 [hep-ph]].

[54] J. -J. Cao, Z. -X. Heng, J. M. Yang, Y. -M. Zhang and J. -Y. Zhu, JHEP 1203, 086 (2012) [arXiv:1202.5821 [hep-ph]].

[55] U. Ellwanger and C. Hugonie, Adv. High Energy Phys. 2012, 625389 (2012) [arXiv:1203.5048 [hep-ph]].

[56] C. Beskidt, W. de Boer and D. I. Kazakov, Phys. Lett. B 726, 758 (2013) [arXiv:1308.1333 [hep-ph]]. 